\title{
Non-degenerate solutions of universal Whitham hierarchy
}

\author{
Kanehisa Takasaki \\ Graduate School of Human and Environmental Studies, \\ Kyoto University, \\ Yoshida, Sakyo, Kyoto, 606-8501, Japan \\ Takashi Takebe \\ Faculty of Mathematics, \\ State University - Higher School of Economics, \\ Vavilova Street, 7, Moscow, 117312, Russia \\ Lee Peng Teo \\ Department of Applied Mathematics, Faculty of Engineering, \\ University of Nottingham Malaysia Campus, Jalan Broga, \\ 43500, Semenyih, Selangor Darul Ehsan, Malaysia.
}

\begin{abstract}
The notion of non-degenerate solutions for the dispersionless Toda hierarchy is generalized to the universal Whitham hierarchy of genus zero with $M+1$ marked points. These solutions are characterized by a Riemann-Hilbert problem (generalized string equations) with respect to two-dimensional canonical transformations, and may be thought of as a kind of general solutions of the hierarchy. The Riemann-Hilbert problem contains $M$ arbitrary functions $H_{a}\left(z_{0}, z_{a}\right), a=1, \ldots, M$, which play the role of generating functions of two-dimensional canonical transformations. The solution of the Riemann-Hilbert problem is described by period maps on the space of $(M+1)$-tuples $\left(z_{\alpha}(p): \alpha=\right.$ $0,1, \ldots, M)$ of conformal maps from $M$ disks of the Riemann sphere and their complements to the Riemann sphere. The period maps are defined by an infinite number of contour integrals that generalize the notion of harmonic moments. The $F$-function (free energy) of these solutions is also shown to have a contour integral representation.
\end{abstract}




\section{Introduction}

The universal Whitham hierarchy is a unified framework for various dispersionless integrable systems and Whitham modulation equations [4]. In particular, the hierarchy of genus zero, which is the subject of this paper, is a natural generalization of the dispersionless KP and Toda hierarchies [9]. Therefore it is natural to ask to what extent the rich contents of the dispersionless KP and Toda hierarchies can be generalized to the hierarchy of genus zero.

This issue has been sought for since the turn of the century when the study of dispersionlss integrable systems entered a new stage. As regards the problem of special solutions, the classical "hodograph method" has been generalized [1, 11] to obtain a class of solutions including Krichever's "algebraic orbits" [4. Another class of special solutions (also related to algebraic orbits) have been studied in the context of the Virasoro constraints [5, 6] and the large- $N$ limit of multiple orthogonal polynomials [7]. It should be stressed that the structure of infinitesimal additional symmetries (including the Virasoro symmetries) was fully elucidated by the work of the Madrid group [5, 6]. As in the case of the dispersionless KP and Toda hierarchies [9], those symmetries are derived from a "nonlinear" Riemann-Hilbert problem

(or an equivalent $\bar{\partial}$ problem [2, 3]) with respect to two-dimensional canonical transformations. As regards the Riemann-Hilbert problem itself, however, no effective method for finding an explicit form of solutions is known apart from very special cases; one has to resort to a genuine existence theorem (though it is enough for deriving the infinitesimal symmetries). Moreover, the $F$-function (free energy), also known as the dispersionless (logarithm of) tau function, has to be treated separately in this approach.

Recently, one of the present authors reformulated the Riemann-Hilbert problem for the dispersionless Toda hierarchy in a slightly different form, and introduced the notion of "non-degenerate solutions" for which a more effective description is available [13. A central idea of this result stems from the work of Wiegmann and Zabrodin [14] on an integrable structure of univalent conformal maps in Riemann's mapping theorem. They used the harmonic moments of the domain to interpret the conformal maps as a special solution of the dispersionless Toda hierarchy. This result can be generalized to pairs of conformal maps [12]. The harmonic moments are redefined therein as contour integrals that include the conformal map (or the pair of conformal maps), and shown to give a system of local coordinates on the space of pairs of conformal maps. Actually, this amounts to solving a Riemann-Hilbert problem (or "string equations") in a special case [8]. 
The method of harmonic moments were generalized later by Zabrodin to a larger class of solutions of the dispersionless Toda hierarchy [15]. The notion of non-degenerate solutions is a rigorous reformulation of those solutions, which thereby turn out to be a kind of general (or generic) solutions of the dispersionless Toda hierarchy. The goal of this paper is to generalize these results [13] to the universal Whitham hierarchy of genus zero.

Let us briefly recall the notion of non-degenerate solutions of the dispersionless Toda hierarchy. Those solutions are characterized by a RiemannHilbert problem of the following form: Let $H(z, \tilde{z})$ be a holomorphic function of two variables defined in a suitable domain (not specified here), and $H_{z}(z, \tilde{z})$ and $H_{\tilde{z}}(z, \tilde{z})$ denote the derivatives $H_{z}(z, \tilde{z})=\partial H(z, \tilde{z}) / \partial z$, $H_{\tilde{z}}(z, \tilde{z})=\partial H(z, \tilde{z}) / \partial \tilde{z}$. Moreover, suppose that $H(z, \tilde{z})$ satisfies the nondegeneracy condition

$$
H_{z \tilde{z}}(z, \tilde{z}) \neq 0 .
$$

The problem is to find four functions $\mathcal{L}(P), \mathcal{M}(P), \tilde{\mathcal{L}}(P), \tilde{\mathcal{M}}(P)$ of a complex variable $P$ with the following properties:

(i) $\mathcal{L}(P)$ and $\mathcal{M}(P)$ are holomorphic functions in the punctured disk $1<$ $|P|<\infty, \mathcal{L}(P)$ being univalent therein, and have a Laurent expansion of the form

$$
\begin{aligned}
\mathcal{L}(P) & =P+\sum_{n=1}^{\infty} u_{n} P^{-n+1} \\
\mathcal{M}(P) & =\sum_{n=1}^{\infty} n t_{n} \mathcal{L}(P)^{n}+t_{0}+\sum_{n=1}^{\infty} v_{n} \mathcal{L}(P)^{n} .
\end{aligned}
$$

(ii) $\tilde{\mathcal{L}}(P)^{-1}$ and $\tilde{\mathcal{M}}(P)$ are holomorphic functions in the punctured disk $0<|P|<1, \tilde{\mathcal{L}}(P)$ being univalent therein, and have a Laurent expansion of the form

$$
\begin{aligned}
\tilde{\mathcal{L}}(P)^{-1} & =\sum_{n=0}^{\infty} \tilde{u}_{n} P^{n-1} \quad\left(\tilde{u}_{0} \neq 0\right), \\
\tilde{\mathcal{M}}(P) & =-\sum_{n=1}^{\infty} n t_{-n} \tilde{\mathcal{L}}(P)^{-n}+t_{0}-\sum_{n=1}^{\infty} v_{-n} \tilde{\mathcal{L}}(P)^{n} .
\end{aligned}
$$

(iii) These functions can be analytically continued to a neighborhood of the unit circle $|P|=1$ and satisfy the functional equations (generalized string equations)

$$
\mathcal{M}(P)=\mathcal{L}(P) H_{z}(\mathcal{L}(P), \tilde{\mathcal{L}}(P)), \quad \tilde{\mathcal{M}}(P)=-\tilde{\mathcal{L}}(P) H_{\tilde{z}}(\mathcal{L}(P), \tilde{\mathcal{L}}(P))
$$


therein.

If the equations

$$
w=z H_{z}(z, \tilde{z}), \quad \tilde{w}=-\tilde{z} H_{\tilde{z}}(z, \tilde{z})
$$

can be solved for $\tilde{z}$, the map $(z, w) \mapsto(f(z, w), g(z, w))=(\tilde{z}, \tilde{w})$ becomes a two-dimensional canonical transformation (or symplectic map) with respect to the symplectic form

$$
\frac{d z \wedge d w}{z}=\frac{d \tilde{z} \wedge d \tilde{w}}{\tilde{z}}
$$

the function $H(z, \tilde{z})$ being its "generating function". It is well known that this is a normal form of canonical transformations in a "general position" of the set of all canonical transformations. (1) can be thus rewritten as

$$
\tilde{\mathcal{L}}(P)=f(\mathcal{L}(P), \mathcal{M}(P)), \quad \tilde{\mathcal{M}}(P)=g(\mathcal{L}(P), \mathcal{M}(P)) .
$$

This is a Riemann-Hilbert problem of the standard form that characterizes the Lax and Orlov-Schulman functions of the dispersionless Toda hierarchy 9]. The aforementioned remark on canonical transformations with generating functions imply that the non-degenerate solutions are indeed general solutions of the dispersionless Toda hierarchy.

An advantage of (11) over (2) is that it is "solvable" in the following sense. The generalized string equations (11) can be converted to the infinite system of equations

$$
\begin{aligned}
n t_{n} & =\frac{1}{2 \pi i} \oint_{|P|=1} H_{z}(\mathcal{L}(P), \tilde{\mathcal{L}}(P)) \mathcal{L}(P)^{-n} d \mathcal{L}(P), \\
n t_{-n} & =\frac{1}{2 \pi i} \oint_{|P|=1} H_{\tilde{z}}(\mathcal{L}(P), \tilde{\mathcal{L}}(P)) \tilde{\mathcal{L}}(P)^{n} d \tilde{\mathcal{L}}(P) \\
t_{0} & =\frac{1}{2 \pi i} \oint_{|P|=1} H_{z}(\mathcal{L}(P), \tilde{\mathcal{L}}(P)) d \mathcal{L}(P) \\
& =-\frac{1}{2 \pi i} \oint_{|P|=1} H_{\tilde{z}}(\mathcal{L}(P), \tilde{\mathcal{L}}(P)) d \tilde{\mathcal{L}}(P)
\end{aligned}
$$

and

$$
\begin{aligned}
v_{n} & =\frac{1}{2 \pi i} \oint_{|P|=1} H_{z}(\mathcal{L}(P), \tilde{\mathcal{L}}(P)) \mathcal{L}(P)^{n} d \mathcal{L}(P), \\
v_{-n} & =\frac{1}{2 \pi i} \oint_{|P|=1} H_{\tilde{z}}(\mathcal{L}(P), \tilde{\mathcal{L}}(P)) \tilde{\mathcal{L}}(P)^{-n} d \tilde{\mathcal{L}}(P)
\end{aligned}
$$


for $n=1,2, \ldots$. Note that the contour integrals are analogues of harmonic moments; in the terminology of geometry, they are a kind of "period integrals". A fundamental fact [13] is that the first set (3) of these period integrals give a system of local coordinates on the space of the pairs $(\mathcal{L}, \tilde{\mathcal{L}})$ of conformal maps. This implies that the "period map" $(\mathcal{L}, \tilde{\mathcal{L}}) \mapsto\left(t_{n}: n \in \mathbf{Z}\right)$ is (locally) invertible, and the inverse map and the second set (4) of period integrals give a (unique) solution of the Riemann-Hilbert problem. Remarkably, the $F$-function, too, turns out to have a contour integral representation [13].

In the language of the universal Whitham hierarchy of genus zero, the dispersionless Toda hierarchy amounts to the case with two "marked points". The general $(M+1)$-point hierarchy is formulated by $M+1$ pairs $\left(z_{\alpha}(p), \zeta_{\alpha}(p)\right)$, $\alpha=0,1, \ldots, M$, of Lax and Orlov-Schulman functions. In the two-point $(M=1)$ case, these functions are connected with the Lax and OrlovSchulman functions of the dispersionless Toda hierarchy as

$$
\begin{array}{rlrl}
z_{0}(p) & =\mathcal{L}(P), & z_{1}(p)=\tilde{\mathcal{L}}(P)^{-1}, \\
\zeta_{0}(p)=\mathcal{M}(P) \mathcal{L}(P)^{-1}, & \zeta_{1}(p)=-\tilde{\mathcal{M}}(P) \tilde{\mathcal{L}}(P),
\end{array}
$$

where the coordinates $p$ and $P$ of the Riemann sphere in both hierarchies are related as

$$
p=P+u_{1}
$$

Thus the marked points $P=\infty, 0$ of the dispersionless Toda hierarchy correspond to the marked points $p=\infty, u_{1}$ of the universal Whitham hierarchy. Bearing this interpretation of the dispersionless Toda hierarchy in mind, we turn to the $M+1$-point case.

This paper is organized as follows. In Section 2, we review the fundamental structure of the universal Whitham hierarchy of genus zero. Building blocks of the hierarchy, such as the Lax and Orlov-Schulmann functions, the $S$-functions, the $F$-function and the generalized Grunsky coefficients, are introduced in detail. For technical reasons, the definition of the $F$-function in our previous work [10, 11] is slightly modified here, though this is not a serious problem. In Section 3, we formulate the Riemann-Hilbert problem that defines non-degenerate solutions. The basic setup is parallel to the formulation by the Madrid group [5, 6]. Our generalized string equations have $M$ arbitrary functions $H_{a}\left(z_{0}, z_{a}\right), a=1, \ldots, M$, as functional data. As in the case of the dispersionless Toda hierarchy, these functions play the role of generating functions of two-dimensional canonical transformations. In Section 4, we generalize the period integrals (3) and (4) to the space $\mathcal{Z}$ 
of $(M+1)$-tuples $\left(z_{\alpha}(p): \alpha=0,1, \ldots, M\right)$ of conformal maps, and show that a half of them give a system of local coordinates on $\mathcal{Z}$. This justifies the definition of non-degenerate solutions. Section 5 is an intermediate step towards the construction of the $F$-function. We present here a contour integral representation of the potentials $\phi_{a}, a=1, \ldots, M$, that show up in the Laurent expansions of the $S$-functions. These $\phi$-functions are used in Section 6 for the construction of the $F$-function. As in the case of the dispersionless Toda hierarchy, we define a set of auxiliary functions $J_{a, 1}\left(z_{0}, z_{a}\right), J_{a, 2}\left(z_{0}, z_{a}\right), a=1, \ldots, M$. These functions are used to express the $F$-function in terms of contour integrals. In Section 7 , we illustrate the construction of non-degenerate solutions in a few special cases that amount to the examples studied for the dispersionless Toda hierarchy [13].

Acknowledgements This work is partly supported by Grant-in-Aid for Scientific Research No. 19104002, 19540179 and No. 21540218 from the Japan Society for the Promotionof Science. TT is partly supported by the grant of the State University - Higher School of Economics, Russia, for the Individual Research Project 09-01-0047 (2009).

\section{Building blocks of universal Whitham hierarchy}

In this section we review essential facts on the universal Whitham hierarchy of genus zero necessary for our later discussion, following our previous work [10, 11]1. The notations are mostly the same as [10, 11], except that, after the notation of the recent work [7] of the Madrid group, Greek indices $\alpha, \beta, \ldots$ range over $0,1, \ldots, M$ and Latin indices $a, b, \ldots$ over $1, \ldots, M$.

Lax functions The Lax functions $z_{\alpha}(p), \alpha=0,1, \ldots, M$, are functions with Laurent expansions of the form

$$
\begin{aligned}
& z_{0}(p)=p+\sum_{j=2}^{\infty} u_{0 j} p^{-j+1} \\
& z_{a}(p)=\frac{r_{a}}{p-q_{a}}+\sum_{j=1}^{\infty} u_{a j}\left(p-q_{a}\right)^{j-1} \quad(a=1, \ldots, M),
\end{aligned}
$$

\footnotetext{
${ }^{1}$ The authors of [10] sincerely apologize numerous typographical errors in the proofs in it, but the statements there are correct. The only differences from [10] are the definition of the $F$-function (29) and, consequently, changes of several signatures in, e.g., (32).
} 
in a neighborhood of $p=\infty$ and $p=q_{a}$, respectively. The coefficients $u_{\alpha j}\left(r_{a}=u_{a 0}\right)$ and the centers $q_{a}$ are dynamical variables. To consider a Riemann-Hilbert problem [5, 6], we choose a set of disjoint positively oriented simple closed curves $C_{1}, \ldots, C_{M}$ that encircle $q_{1}, \ldots, q_{M}$ counterclockwise, and assume that the Laurent expansion of $z_{a}(p)$ converges in the inside $D_{a}$ of $C_{a}$ and that the Laurent expansion of $z_{0}(p)$ converges in a neighborhood of $p=\infty$ and can be analytically continued, as a holomorphic function, to the outside $\mathbf{C} \backslash\left(D_{1} \cup \cdots \cup D_{M}\right)$ of $D_{a}$ 's.

Lax equations The hierarchy has $M+1$ series of time evolutions with time variables $t_{0 n}, n=1,2, \ldots$ and $t_{a n}, a=1, \ldots, M, n=0,1,2, \ldots$ The time evolutions of the Lax functions are defined by the Lax equations

$$
\partial_{\alpha n} z_{\beta}(p)=\left\{\Omega_{\alpha n}(p), z_{\beta}(p)\right\}, \quad \partial_{\alpha n}=\partial / \partial t_{\alpha n},
$$

with respect to the Poisson bracket

$$
\{f, g\}=\frac{\partial f}{\partial p} \frac{\partial g}{\partial t_{01}}-\frac{\partial f}{\partial t_{01}} \frac{\partial g}{\partial p} .
$$

The Hamiltonians $\Omega_{\alpha n}(p)$ are defined as

$$
\begin{aligned}
& \Omega_{0 n}(p)=\left(z_{0}(p)^{n}\right)_{(0, \geq 0)}, \quad \Omega_{a n}(p)=\left(z_{a}(p)^{n}\right)_{(a,<0)} \quad(n=1,2, \ldots), \\
& \Omega_{a 0}(p)=-\log \left(p-q_{a}\right),
\end{aligned}
$$

where ()$_{(0, \geq 0)}$ denotes the projection to non-negative powers of $p$, and ()$_{(a,<0)}$ the projection to negative powers of $p-q_{a}$. In other words,

$$
\begin{aligned}
& z_{0}(p)^{n}=\Omega_{0 n}(p)+O\left(p^{-1}\right) \quad(p \rightarrow \infty), \\
& z_{a}(p)^{n}=\Omega_{a n}(p)+O(1) \quad\left(p \rightarrow q_{a}\right)
\end{aligned}
$$

for $n \geq 1$. $\Omega_{\alpha n}(p)$ satisfies the dispersionless Zakharov-Shabat equations

$$
\partial_{\beta m} \Omega_{\alpha n}(p)-\partial_{\alpha n} \Omega_{\beta m}(p)+\left\{\Omega_{\alpha n}(p), \Omega_{\beta m}(p)\right\}=0 .
$$

As pointed out in [5], the dressing functions of the universal Whitham hierarchy have the following form:

$$
\begin{gathered}
\varphi_{0}(p)=\sum_{j=1}^{\infty} \varphi_{0, j} p^{-j}, \quad \varphi_{a}(p)=\sum_{j=0}^{\infty} \varphi_{a, j}\left(p-q_{a}^{(0)}\right)^{j}, \\
z_{0}(p)=e^{\operatorname{ad} \varphi_{0}(p)} p, \quad z_{a}(p)=e^{\operatorname{ad} \varphi_{a}(p)}\left(p-q_{a}^{(0)}\right)^{-1} .
\end{gathered}
$$

The following is due to [5], Theorem3. 
Proposition 2.1. If $\left(z_{\alpha}(p): \alpha=0,1, \ldots, M\right)$ is a solution of the universal Whitham hierarchy, then there exists dressing functions $\varphi_{\alpha}(p)$ of the form (111), such that

$$
z_{0}(p)=e^{a d \varphi_{0}(p)} p, \quad z_{a}(p)=e^{a d \varphi_{a}(p)}\left(p-q_{a}^{(0)}\right)^{-1},
$$

and

$$
\nabla_{\alpha n} \varphi_{\beta}=\tilde{\Omega}_{\alpha n, \beta},
$$

where

$$
\tilde{\Omega}_{\alpha n, \beta}= \begin{cases}\Omega_{\alpha n}-\delta_{\alpha 0} \delta_{n 1} z_{\beta}(p)^{-1} & (\alpha \neq \beta \text { and }(\beta \neq 0 \text { or } n \neq 0)), \\ \Omega_{\alpha 0}+\log z_{0}(p) & (\alpha \neq 0 \text { and } \beta=0 \text { and } n=0), \\ \Omega_{\alpha n}-z_{\alpha}(p)^{n} & (\alpha=\beta \text { and } n \neq 0), \\ \Omega_{\alpha 0}-\log z_{\alpha}(p) & (\alpha=\beta \neq 0 \text { and } n=0),\end{cases}
$$

and $\nabla_{\alpha n}$ is the right logarithmic derivative (cf. [5] Appendix A, [9] Appendix A) defined by

$$
\nabla_{\alpha n} \psi=\sum_{n=0}^{\infty} \frac{(\mathrm{ad} \psi)^{n}}{(n+1) !} \partial_{\alpha n} \psi
$$

In the above, $q_{a}^{(0)}, a=1, \ldots, M$, are arbitrary non-dynamical variables. Without loss of generality, we set $q_{a}^{(0)}=0$ henceforth.

Orlov-Schulman functions The Orlov-Schulman functions $\zeta_{\alpha}(p), \alpha=$ $0,1, \ldots, M$ are Laurent series of the form

$$
\begin{aligned}
& \zeta_{0}(p)=\sum_{n=1}^{\infty} n t_{0 n} z_{0}(p)^{n-1}+\frac{t_{00}}{z_{0}(p)}+\sum_{n=1}^{\infty} z_{0}(p)^{-n-1} v_{0 n}, \\
& \zeta_{a}(p)=\sum_{n=1}^{\infty} n t_{a n} z_{a}(p)^{n-1}+\frac{t_{a 0}}{z_{a}(p)}+\sum_{n=1}^{\infty} z_{a}(p)^{-n-1} v_{a n},
\end{aligned}
$$

where

$$
t_{00}=-\sum_{a=1}^{M} t_{a 0} .
$$

They satisfy the Lax equations

$$
\partial_{\alpha n} \zeta_{\beta}(p)=\left\{\Omega_{\alpha n}(p), \zeta_{\beta}(p)\right\}
$$


and the canonical Poisson commutation relation

$$
\left\{z_{\alpha}(p), \zeta_{\alpha}(p)\right\}=1 .
$$

In terms of the dressing functions, $\zeta_{\alpha}$ are given by

$$
\begin{aligned}
& \zeta_{0}(p)=e^{\operatorname{ad} \varphi_{0}(p)}\left(\sum_{n=1}^{\infty} n t_{0 n} p^{n-1}+\frac{t_{00}}{p}\right), \\
& \zeta_{a}(p)=e^{\operatorname{ad} \varphi_{a}(p)}\left(\sum_{n=1}^{\infty} n t_{a n} p^{-n+1}+t_{a 0} p-t_{01} p^{2}\right) .
\end{aligned}
$$

The canonical Poisson commutation relation (18) is a direct consequence of the definition and the Lax equations (17) follow from (14).

$S$-functions The $S$-functions $\mathcal{S}_{\alpha}(p), \alpha=0,1, \ldots, M$, are defined as potentials of 1 -forms as

$$
d \mathcal{S}_{\alpha}(p)=\theta+\zeta_{\alpha}(p) d z_{\alpha}(p),
$$

where

$$
\theta=\sum_{n=1}^{\infty} \Omega_{0 n}(p) d t_{0 n}+\sum_{a=1}^{M} \sum_{n=0}^{\infty} \Omega_{a n}(p) d t_{a n} .
$$

They have Laurent expansions of the form

$$
\begin{aligned}
& \mathcal{S}_{0}(p)=\sum_{n=1}^{\infty} t_{0 n} z_{0}(p)^{n}+t_{00} \log z_{0}(p)-\sum_{n=1}^{\infty} \frac{z_{0}(p)^{-n}}{n} v_{0 n} \\
& \mathcal{S}_{a}(p)=\sum_{n=1}^{\infty} t_{a n} z_{a}(p)^{n}+t_{a 0} \log z_{a}(p)+\phi_{a}-\sum_{n=1}^{\infty} \frac{z_{a}(p)^{-n}}{n} v_{a n} .
\end{aligned}
$$

Implications of $S$-functions Let us define $S_{\alpha}(z), \alpha=0,1, \ldots, M$, as

$$
\begin{aligned}
& S_{0}(z)=\sum_{n=1}^{\infty} t_{0 n} z^{n}+t_{00} \log z-\sum_{n=1}^{\infty} \frac{z^{-n}}{n} v_{0 n}, \\
& S_{a}(z)=\sum_{n=1}^{\infty} t_{a n} z^{n}+t_{a 0} \log z+\phi_{a}-\sum_{n=1}^{\infty} \frac{z^{-n}}{n} v_{a n} .
\end{aligned}
$$

$\mathcal{S}_{\alpha}(p)$ can be thereby expressed as

$$
\mathcal{S}_{0}(p)=S_{0}\left(z_{0}(p)\right), \quad \mathcal{S}_{a}(p)=S_{a}\left(z_{a}(p)\right) .
$$


Moreover, the defining equations of $\mathcal{S}_{\alpha}(p)$ imply the equations

$$
\zeta_{\alpha}(p)=S_{\alpha}^{\prime}\left(z_{\alpha}(p)\right),
$$

where the prime denotes the derivative with respect to $z$, and

$$
\Omega_{\alpha n}(p)=\left.\partial_{\alpha n} S_{\beta}(z)\right|_{z=z_{\beta}(p)}, \quad \beta=0,1, \ldots, M .
$$

The former is just a restatement of the Laurent expansion of $\zeta_{\alpha}(p)$. The latter implies that $\Omega_{\alpha n}(p)$ can be written in several different forms as

$$
\begin{aligned}
& \Omega_{0 n}(p)=\left\{\begin{array}{l}
z_{0}(p)^{n}-\sum_{m=1}^{\infty} \frac{z_{0}(p)^{-m}}{m} \partial_{0 n} v_{0 m}, \\
\partial_{0 n} \phi_{b}-\sum_{m=1}^{\infty} \frac{z_{b}(p)^{-m}}{m} \partial_{0 n} v_{b m}, \quad b=1, \ldots, M
\end{array}\right. \\
& \Omega_{a n}(p)=\left\{\begin{array}{l}
-\sum_{m=1}^{\infty} \frac{z_{0}(p)^{-m}}{m} \partial_{a n} v_{0 m}, \\
\delta_{a b} z_{b}(p)^{n}+\partial_{a n} \phi_{b}-\sum_{m=1}^{\infty} \frac{z_{b}(p)^{-m}}{m} \partial_{a n} v_{b m}, \quad b=1, \ldots, M
\end{array}\right.
\end{aligned}
$$

for $n=1,2, \ldots$, and

$$
\Omega_{a 0}(p)=\left\{\begin{array}{l}
-\log z_{0}(p)-\sum_{m=1}^{\infty} \frac{z_{0}(p)^{-m}}{m} \partial_{a 0} v_{0 m}, \\
\delta_{a b} \log z_{b}(p)+\partial_{a 0} \phi_{b}-\sum_{m=1}^{\infty} \frac{z_{b}(p)^{-m}}{m} \partial_{a 0} v_{b m}, \quad b=1, \ldots, M .
\end{array}\right.
$$

In particular, since $\Omega_{01}(p)=p$, we have the identities

$$
\begin{aligned}
& p=z_{0}(p)-\sum_{m=1}^{\infty} \frac{z_{0}(p)^{-m}}{m} \partial_{01} v_{0 m}, \\
& p=\partial_{01} \phi_{b}-\sum_{m=1}^{\infty} \frac{z_{b}(p)^{-m}}{m} \partial_{01} v_{b m}, \quad b=1, \ldots, M,
\end{aligned}
$$

which imply that the inverse functions $p=p_{0}(z)$ and $p=p_{b}(z)$ of $z=z_{0}(p)$ and $z=z_{b}(p)$ are given explicitly by

$$
\begin{array}{r}
p_{0}(z)=z-\sum_{m=1}^{\infty} \frac{z^{-m}}{m} \partial_{01} v_{0 m}=\partial_{01} S_{0}(z), \\
p_{b}(z)=\partial_{01} \phi_{b}-\sum_{m=1}^{\infty} \frac{z^{-m}}{m} \partial_{01} v_{b m}=\partial_{01} S_{b}(z) .
\end{array}
$$


Consequently,

$$
q_{a}=\partial_{01} \phi_{a}, \quad r_{a}=-\partial_{01} v_{a 1}
$$

Substituting $p=p_{\beta}(z)$ in

$$
\left.\partial_{\alpha n} S_{\beta}(z)\right|_{z=z_{\beta}(p)}=\Omega_{\alpha n}(p)
$$

leads to the Hamilton-Jacobi equations

$$
\partial_{\alpha n} S_{\beta}(z)=\Omega_{\alpha n}\left(\partial_{01} S_{\beta}(z)\right) .
$$

$F$-function The $F$-function is defined by the equation

$$
\begin{aligned}
& \partial_{0 n} F=v_{0 n}, \quad \partial_{a n} F=v_{a n}, \quad n=1,2, \ldots, \\
& \partial_{a 0} F=-\phi_{a}+\sum_{b=1}^{a-1} t_{b 0} \log (-1), \quad a=1, \ldots, M .
\end{aligned}
$$

The last part containing $\log (-1)$ is slightly different from the definition of the Madrid group [6, 5] and the previous paper [10] of the first two authors, but this is due to arbitrariness of the $F$-function. With the $F$-function, the $S$-functions can be written as

$$
\begin{aligned}
& S_{0}(z)=\sum_{n=1}^{\infty} t_{0 n} z^{n}+t_{00} \log z-D_{0}(z) F \\
& S_{a}(z)=\sum_{n=1}^{\infty} t_{a n} z^{n}+t_{a 0} \log z+\phi_{a}-D_{a}(z) F,
\end{aligned}
$$

where $D_{0}(z)$ and $D_{a}(z)$ denote the following differential operators:

$$
D_{0}(z)=\sum_{n=1}^{\infty} \frac{z^{-n}}{n} \partial_{0 n}, \quad D_{a}(z)=\sum_{n=1}^{\infty} \frac{z^{-n}}{n} \partial_{a n} .
$$

Generalized Faber polynomials and Grunsky coefficients The Hamiltonians $\Omega_{\alpha n}(p)$ of the Lax equations can also be characterized by the generating functions

$$
\begin{aligned}
& \log \frac{p_{0}(z)-q}{z}=-\sum_{n=1}^{\infty} \frac{z^{-n}}{n} \Omega_{0 n}(q), \\
& \log \frac{q-p_{a}(z)}{q-q_{a}}=-\sum_{n=1}^{\infty} \frac{z^{-n}}{n} \Omega_{a n}(q) .
\end{aligned}
$$


The left hand sides of these identities are understood to be rewritten

$$
\log \frac{p_{0}(z)-q}{z}=\log \frac{p_{0}(z)}{z}+\log \left(1-\frac{q}{p_{0}(z)}\right)
$$

and

$$
\log \frac{q-p_{a}(z)}{q-q_{a}}=\log \left(1-\frac{p_{a}(z)-q_{a}}{q-q_{a}}\right)
$$

and expanded to power series of $q$ and $\left(q-q_{a}\right)^{-1}$, respectively.

The generalized Grunsky coefficients $b_{a m b n}=b_{\text {bnam }}$ are defined by the generating functions

$$
\begin{aligned}
\log \frac{p_{0}(z)-p_{0}(w)}{z-w} & =-\sum_{m, n=1}^{\infty} z^{-m} w^{-n} b_{0 m 0 n}, \\
\log \frac{p_{0}(z)-p_{a}(w)}{z} & =-\sum_{m=1}^{\infty} \sum_{n=0}^{\infty} z^{-m} w^{-n} b_{0 m a n}, \\
\log \frac{z w\left(p_{a}(z)-p_{a}(w)\right)}{w-z} & =-\sum_{m, n=0}^{\infty} z^{-m} w^{-n} b_{a m a n}, \\
\log \frac{p_{a}(z)-p_{b}(w)}{\epsilon_{a b}} & =-\sum_{m, n=0}^{\infty} z^{-m} w^{-n} b_{a m b n} \quad(a \neq b) .
\end{aligned}
$$

They are related to the $F$-function as

$$
\hat{\partial}_{\alpha m} \hat{\partial}_{\beta n} F=-b_{\alpha m \beta n} \quad(\alpha, \beta=0,1, \ldots, N),
$$

where

$$
\epsilon_{a b}=\left\{\begin{array}{ll}
+1 & (a \leq b) \\
-1 & (a>b)
\end{array}, \quad \hat{\partial}_{\alpha n}= \begin{cases}\frac{1}{n} \partial_{\alpha n} & (n \neq 0) \\
\partial_{\alpha 0} & (n=0)\end{cases}\right.
$$

\section{Riemann-Hilbert problem and non-degenerate solutions}

Following the work of the Madrid group [ 5 , 6], we now formulate a RiemannHilbert problem. Choose a set of positively oriented simple closed curves $C_{1}, \ldots, C_{M}$ and let $D_{1}, \ldots, D_{M}$ denote their inside domains. The RiemannHilbert data consist of $M$ pairs $\left(f_{a}, g_{a}\right), a=1, \ldots, M$, of holomorphic functions $f_{a}=f_{a}\left(p, t_{01}\right), g_{a}=g_{a}\left(p, t_{01}\right)$ of $p, t_{01}$ (defined in a suitable domain) 
that satisfy the conditions

$$
\left\{f_{a}, g_{a}\right\}=\frac{\partial f_{a}}{\partial p} \frac{\partial g_{a}}{\partial t_{01}}-\frac{\partial f_{a}}{\partial t_{01}} \frac{\partial g_{a}}{\partial p}=1
$$

thus defining two-dimensional canonical transformations. The problem is to seek $M+1$ pairs $\left(z_{\alpha}(p), \zeta_{\alpha}(p)\right), \alpha=0,1, \ldots, M$, of functions of $p$ and $\boldsymbol{t}=\left\{t_{0 n}: n=1,2, \ldots\right\} \cup\left\{t_{a n}: a=1, \ldots, M, n=0,1,2, \ldots\right\}$ that satisfy the following conditions:

(i) $z_{0}(p)$ and $\zeta_{0}(p)$ are holomorphic functions on $\mathbf{C} \backslash\left(D_{1} \cup \ldots \cup D_{M}\right)$, $z_{0}(p)$ is univalent therein (in particular, $z_{0}^{\prime}(p)$ does not vanish) and, as $p \rightarrow \infty$,

$$
\begin{aligned}
& z_{0}(p)=p+O\left(p^{-1}\right), \\
& \zeta_{0}(p)=\sum_{n=1}^{\infty} n t_{0 n} z_{0}(p)^{n-1}+\frac{t_{00}}{z_{0}(p)}+O\left(p^{-2}\right) .
\end{aligned}
$$

(ii) $z_{a}(p)$ and $\zeta_{a}(p)$ are holomorphic functions on $D_{a}$ punctured at a point $q_{a} \in D_{a}, z_{a}^{-1}(p)$ is univalent on $D_{a}$ and, as $p \rightarrow q_{a}$,

$$
\begin{aligned}
& z_{a}(p)=\frac{r_{a}}{p-q_{a}}+O(1), \\
& \zeta_{a}(p)=\sum_{n=1}^{\infty} n t_{a n} z_{a}(p)^{n-1}+\frac{t_{a 0}}{z_{a}(p)}+O\left(\left(p-q_{a}\right)^{2}\right) .
\end{aligned}
$$

$q_{a}$ and $r_{a}$ are functions of the time variables to be thus determined.

(iii) For $a=1, \ldots, M$, the four functions $z_{0}(p), \zeta_{0}(p), z_{a}(p), \zeta_{a}(p)$ can be analytically continued to a neighborhood of $C_{a}$ and satisfy the functional equations

$$
z_{a}(p)=f_{a}\left(z_{0}(p), \zeta_{0}(p)\right), \quad \zeta_{a}(p)=g_{a}\left(z_{0}(p), \zeta_{0}(p)\right)
$$

therein.

Functions $z_{\alpha}(p)$ satisfying above conditions are solutions of the universal Whitham hierarchy and $\zeta_{\alpha}(p)$ 's are corresponding Orlov-Schulman functions, as is proved in [6], Theorem 1.

Note that formally we can prove the converse. Namely there exist Riemann-Hilbert data for each solution of the universal Whitham hierarhcy. 
Proposition 3.1. Let $\left(z_{\alpha}(p): \alpha=0,1, \ldots, M\right)$ be a solution of the universal Whitham hierarchy, and $\left(\zeta_{\alpha}(p): \alpha=0,1, \ldots, M\right)$ the corresponding Orlov-Schulman functions. For $a=1, \ldots, M$, there exist functions $f_{a}\left(p, t_{01}\right)$ and $g_{a}\left(p, t_{01}\right)$ such that

$$
z_{a}=f_{a}\left(z_{0}, \zeta_{0}\right), \quad \zeta_{a}=g_{a}\left(z_{0}, \zeta_{0}\right)
$$

and

$$
\left\{f_{a}\left(p, t_{01}\right), g_{a}\left(p, t_{01}\right)\right\}=1 \text {. }
$$

Proof. This is the same as Propositions 4 and 5 of [5], but let us prove it here in our language as in [9]. Given a solution $\left(z_{\alpha}(p): \alpha=0,1, \ldots, M\right)$ of the universal Whitham hierarchy, construct the dressing functions $\varphi_{\alpha}(p)$ as given by Proposition 2.1. (Recall that we have put $q_{a}^{(0)}=0$.) For any $\alpha$, let

$$
\begin{aligned}
& \tilde{f}_{\alpha}\left(p, t_{01}\right)=\exp \left(-\operatorname{ad} \varphi_{\alpha}(\tilde{\boldsymbol{t}}=0)\right) p, \\
& \tilde{g}_{\alpha}\left(p, t_{01}\right)=\exp \left(-\operatorname{ad} \varphi_{\alpha}(\tilde{\boldsymbol{t}}=0)\right) t_{01}
\end{aligned}
$$

where $\tilde{\boldsymbol{t}}=\boldsymbol{t} \backslash\left\{t_{01}\right\}$. Notice that

$$
\begin{aligned}
z_{0}(p, \tilde{\boldsymbol{t}}=0) & =\exp \left(\operatorname{ad} \varphi_{0}(\tilde{\boldsymbol{t}}=0)\right) p, \\
\zeta_{0}(p, \tilde{\boldsymbol{t}}=0) & =\exp \left(\operatorname{ad} \varphi_{0}(\tilde{\boldsymbol{t}}=0)\right) t_{01}, \\
z_{a}^{-1}(p, \tilde{\boldsymbol{t}}=0) & =\exp \left(\operatorname{ad} \varphi_{a}(\tilde{\boldsymbol{t}}=0)\right) p, \\
\left(-z_{a}^{2} \zeta_{a}\right)(p, \tilde{\boldsymbol{t}}=0) & =\exp \left(\operatorname{ad} \varphi_{a}(\tilde{\boldsymbol{t}}=0)\right) t_{01} .
\end{aligned}
$$

Therefore,

$$
\begin{aligned}
& \tilde{f}_{a}\left(z_{a}^{-1}(p, \tilde{\boldsymbol{t}}=0),\left(-z_{a}^{2} \zeta_{a}\right)(p, \tilde{\boldsymbol{t}}=0)\right) \\
= & \tilde{f}_{0}\left(z_{0}(p, \tilde{\boldsymbol{t}}=0), \zeta_{0}(p, \tilde{\boldsymbol{t}}=0)\right)=p, \\
& \tilde{g}_{a}\left(z_{a}^{-1}(p, \tilde{\boldsymbol{t}}=0),\left(-z_{a}^{2} \zeta_{a}\right)(p, \tilde{\boldsymbol{t}}=0)\right) \\
= & \tilde{g}_{0}\left(z_{0}(p, \tilde{\boldsymbol{t}}=0), \zeta_{0}(p, \tilde{\boldsymbol{t}}=0)\right)=t_{01}
\end{aligned}
$$

for any $a$. Now

$$
\begin{aligned}
\frac{\partial}{\partial t_{\beta n}} \tilde{f}_{0}\left(z_{0}, \zeta_{0}\right) & =\left\{\Omega_{\beta n}, \tilde{f}_{0}\left(z_{0}, \zeta_{0}\right)\right\} \\
\frac{\partial}{\partial t_{\beta n}} \tilde{f}_{a}\left(z_{a}^{-1},-z_{a}^{2} \zeta_{a}\right) & =\left\{\Omega_{\beta n}, \tilde{f}_{a}\left(z_{a}^{-1},-z_{a}^{2} \zeta_{a}\right)\right\},
\end{aligned}
$$


and similarly for $\tilde{g}_{0}\left(z_{0}, \zeta_{0}\right)$ and $\tilde{g}_{a}\left(z_{a}^{-1},-z_{a}^{2} \zeta_{a}\right)$. Therefore,

$$
\begin{gathered}
\left.\frac{\partial}{\partial t_{\beta n}} \tilde{f}_{0}\left(z_{0}, \zeta_{0}\right)\right|_{\tilde{\boldsymbol{t}}=0}=\left.\frac{\partial}{\partial t_{\beta n}} \tilde{f}_{a}\left(z_{a}^{-1},-z_{a}^{2} \zeta_{a}\right)\right|_{\tilde{\boldsymbol{t}}=0}, \\
\left.\frac{\partial}{\partial t_{\beta n}} \tilde{g}_{0}\left(z_{0}, \zeta_{0}\right)\right|_{\tilde{\boldsymbol{t}}=0}=\left.\frac{\partial}{\partial t_{\beta n}} \tilde{g}_{a}\left(z_{a}^{-1},-z_{a}^{2} \zeta_{a}\right)\right|_{\tilde{\boldsymbol{t}}=0} .
\end{gathered}
$$

In the same way, one can show that

$$
\begin{aligned}
& \left.\frac{\partial}{\partial t_{\beta_{k} n_{k}}} \cdots \frac{\partial}{\partial t_{\beta_{1} n_{1}}} \tilde{f}_{0}\left(z_{0}, \zeta_{0}\right)\right|_{\tilde{\boldsymbol{t}}=0}=\left.\frac{\partial}{\partial t_{\beta_{k} n_{k}}} \cdots \frac{\partial}{\partial t_{\beta_{1} n_{1}}} \tilde{f}_{a}\left(z_{a}^{-1},-z_{a}^{2} \zeta_{a}\right)\right|_{\tilde{\boldsymbol{t}}=0}, \\
& \left.\frac{\partial}{\partial t_{\beta_{k} n_{k}}} \cdots \frac{\partial}{\partial t_{\beta_{1} n_{1}}} \tilde{g}_{0}\left(z_{0}, \zeta_{0}\right)\right|_{\tilde{\boldsymbol{t}}=0}=\left.\frac{\partial}{\partial t_{\beta_{k} n_{k}}} \cdots \frac{\partial}{\partial t_{\beta_{1} n_{1}}} \tilde{g}_{a}\left(z_{a}^{-1},-z_{a}^{2} \zeta_{a}\right)\right|_{\tilde{\boldsymbol{t}}=0} .
\end{aligned}
$$

These show that

$$
\tilde{f}_{0}\left(z_{0}, \zeta_{0}\right)=\tilde{f}_{a}\left(z_{a}^{-1},-z_{a}^{2} \zeta_{a}\right) \quad \tilde{g}_{0}\left(z_{0}, \zeta_{0}\right)=\tilde{g}_{a}\left(z_{a}^{-1},-z_{a}^{2} \zeta_{a}\right) .
$$

Notice that by definition,

$$
\left\{\tilde{f}_{0}\left(p, t_{01}\right), \tilde{g}_{0}\left(p, t_{01}\right)\right\}=1, \quad\left\{\tilde{f}_{a}\left(p, t_{01}\right), \tilde{g}_{a}\left(p, t_{01}\right)\right\}=1 .
$$

One can solve the equations

$$
\tilde{f}_{0}\left(p, t_{01}\right)=\tilde{f}_{a}\left(\tilde{p}^{-1},-\tilde{p}^{2} \tilde{t}_{01}\right), \quad \tilde{g}_{0}\left(p, t_{01}\right)=\tilde{g}_{a}\left(\tilde{p}^{-1},-\tilde{p}^{2} \tilde{t}_{01}\right),
$$

for $\tilde{p}$ and $\tilde{t}_{01}$, which gives

$$
\tilde{p}=f_{a}\left(p, t_{01}\right), \quad \tilde{t}_{01}=g_{a}\left(p, t_{01}\right) .
$$

This implies (38). It is also straightforward to show that $\left\{f_{a}, g_{a}\right\}=1$.

We now specialize the Riemann-Hilbert problem to the case where the canonical transformations are defined by generating functions $H_{a}\left(z_{0}, z_{a}\right)$, $a=1, \ldots, M$. The generating functions are assumed to satisfy the nondegeneracy conditions

$$
H_{a, z_{0} z_{a}}\left(z_{0}, z_{a}\right) \neq 0 \text {. }
$$

Accordingly, the functional equations (37) connecting the four functions $z_{0}(p), \zeta_{0}(p), z_{a}(p), \zeta_{a}(p)$ are converted to the generalized string equations

$$
\zeta_{0}(p)=H_{a, z_{0}}\left(z_{0}(p), z_{a}(p)\right), \quad \zeta_{a}(p)=-H_{a, z_{a}}\left(z_{0}(p), z_{a}(p)\right) .
$$


Existence of such generating functions $H_{a}$ under the assumption of nondegeneracy of $f_{a}$ 's:

$$
\frac{\partial f_{a}}{\partial t_{01}} \neq 0
$$

can be proved as in [13], §3.4. In fact, under the assumption (41), we can solve the equation $z_{a}=f_{a}\left(z_{0}, \zeta_{0}\right)$ to obtain

$$
\zeta_{0}=A_{a}\left(z_{0}, z_{a}\right)
$$

Equivalently,

$$
z_{a}=f_{a}\left(z_{0}, A_{a}\left(z_{0}, z_{a}\right)\right)
$$

Define $B_{a}\left(z_{0}, z_{a}\right)$ so that

$$
\zeta_{a}=B_{a}\left(z_{0}, z_{a}\right)=g_{a}\left(z_{0}, A_{a}\left(z_{0}, z_{a}\right)\right) .
$$

Differentiating (42) with respect to $z_{0}$ and $z_{a}$ and (43) with respect to $z_{0}$, we find that

$$
\begin{aligned}
& 0=\frac{\partial f_{a}}{\partial z_{0}}+\frac{\partial f_{a}}{\partial \zeta_{0}} \frac{\partial A_{a}}{\partial z_{0}} \quad \Longrightarrow \quad \frac{\partial A_{a}}{\partial z_{0}}=\frac{-\frac{\partial f_{a}}{\partial z_{0}}}{\frac{\partial f_{a}}{\partial \zeta_{0}}} \\
& 1=\frac{\partial f_{a}}{\partial \zeta_{0}} \frac{\partial A_{a}}{\partial z_{a}} \quad \Longrightarrow \quad \frac{\partial A_{a}}{\partial z_{a}}=\frac{1}{\frac{\partial f_{a}}{\partial \zeta_{0}}}, \\
& \frac{\partial B_{a}}{\partial z_{0}}=\frac{\partial g_{a}}{\partial z_{0}}+\frac{\partial g_{a}}{\partial \zeta_{0}} \frac{\partial A_{a}}{\partial z_{0}} \quad \Longrightarrow \quad \frac{\partial B_{a}}{\partial z_{0}}=\frac{\partial g_{a}}{\partial z_{0}}-\frac{\partial g_{a}}{\partial \zeta_{0}} \frac{\frac{\partial f_{a}}{\partial z_{0}}}{\frac{\partial f_{a}}{\partial \zeta_{0}}}=-\frac{1}{\frac{\partial f_{a}}{\partial \zeta_{0}}}
\end{aligned}
$$

Hence we have $\partial_{z_{a}} A_{a}=-\partial_{z_{0}} B_{a}$, which implies that there exists $H_{a}\left(z_{0}, z_{a}\right)$ satisfying (40) and (39).

Our goal in the following is to solve the generalized string equations (40) in the language of geometry of the space

$$
\mathcal{Z}:=\left\{\left(z_{\alpha}(p): \alpha=0, \ldots, M\right) \mid \text { properties of } z_{\alpha}(p) \text { 's in (i), (ii) }\right\}
$$

of the $M+1$-tuple of functions $z_{\alpha}(p), \alpha=0,1, \ldots, M$. This enables us to understand the universal Whitham hierarchy as a system of integrable commuting flows on $\mathcal{Z}$, just as achieved in the case of the dispersionless Toda hierarchy [13]. 
To this end, we define the functions $t_{0 n}, t_{00}, v_{0 n}(n=1,2, \ldots)$ and $t_{a n}, t_{a 0}, v_{a n}(a=1, \ldots, M, n=1,2, \ldots)$ on $\mathcal{Z}$ as

$$
\begin{aligned}
n t_{0 n} & =\sum_{a=1}^{M} \frac{1}{2 \pi i} \oint_{C_{a}} H_{a, z_{0}}\left(z_{0}(p), z_{a}(p)\right) z_{0}(p)^{-n} d z_{0}(p), \\
t_{00} & =\sum_{a=1}^{M} \frac{1}{2 \pi i} \oint_{C_{a}} H_{a, z_{0}}\left(z_{0}(p), z_{a}(p)\right) d z_{0}(p), \\
v_{0 n} & =\sum_{a=1}^{M} \frac{1}{2 \pi i} \oint_{C_{a}} H_{a, z_{0}}\left(z_{0}(p), z_{a}(p)\right) z_{0}(p)^{n} d z_{0}(p)
\end{aligned}
$$

and

$$
\begin{aligned}
n t_{a n} & =\frac{1}{2 \pi i} \oint_{C_{a}} H_{a, z_{a}}\left(z_{0}(p), z_{a}(p)\right) z_{a}(p)^{-n} d z_{a}(p), \\
t_{a 0} & =\frac{1}{2 \pi i} \oint_{C_{a}} H_{a, z_{a}}\left(z_{0}(p), z_{a}(p)\right) d z_{a}(p), \\
v_{a n} & =\frac{1}{2 \pi i} \oint_{C_{a}} H_{a, z_{a}}\left(z_{0}(p), z_{a}(p)\right) z_{a}(p)^{n} d z_{a}(p) .
\end{aligned}
$$

This is just a restatement of the string equations (40). $t_{00}$ and $t_{a 0}$ 's are automatically constrained as

$$
t_{00}=-\sum_{a=1}^{\infty} t_{a 0} .
$$

The contour integrals on the right hand side of (44) are derived by continuously deforming a simple closed curve $C_{\infty}$ encircling $p=\infty$ and separating it from all $D_{a}$ 's. Notice that since $z_{a}\left(q_{a}\right)=\infty, z_{a}(p)$ maps the inside of $D_{a}$ onto the outside of $z_{a}\left(C_{a}\right)$. Therefore,

$$
\oint_{C_{a}} z_{a}(p)^{m} z_{a}^{\prime}(p) d p=-\delta_{m,-1}
$$

In the next section, we shall reconstruct $\partial_{\alpha n}$ 's as globally defined vector fields on $\mathcal{Z}$, and show that $t_{\alpha n}$ 's may be thought of as "dual" (local) coordinates on $\mathcal{Z}$ with respect to these vector fields. This is the same geometric situation as observed in the case of the dispersionless Toda hierarchy [13]. The universal Whitham hierarchy is thus realized as a system of commuting flows on $\mathcal{Z}$. This geometric setting can be cast into the usual setting in the $\boldsymbol{t}$ space by the inverse of the period map $\left(z_{\alpha}(p): \alpha=0,1, \ldots, M\right) \mapsto \boldsymbol{t}$. The 
functions $z_{\alpha}(p)$ and $\zeta_{\alpha}(p)$ on $\mathcal{Z}$ are pulled back by this inverse period map to become a solution of the string equations (40), hence a solution of the universal Whitham hierarchy.

The $S$-function (in particular $\phi_{a}$ ) and the $F$-function, too, can be primarily defined as a function on $\mathcal{Z}$, then pulled back to the $\boldsymbol{t}$ space. We shall discussed this issue in later sections.

\section{Construction of vector fields $\partial_{\alpha n}$ on $\mathcal{Z}$}

Following [13], we reconstruct $\partial_{\alpha n}$ 's as vector fields on $\mathcal{Z}$.

Theorem 4.1. If the vector fields $\partial_{0 n}(n=1,2, \ldots)$ and $\partial_{a n}(a=1, \ldots, M$, $n=0,1,2, \ldots)$ on $\mathcal{Z}$ satisfy the equations

$$
\frac{\partial_{\alpha n} z_{b}(p)}{z_{b}^{\prime}(p)}-\frac{\partial_{\alpha n} z_{0}(p)}{z_{0}^{\prime}(p)}=\frac{\Omega_{\alpha n}^{\prime}(p)}{z_{0}^{\prime}(p) z_{b}^{\prime}(p) H_{b, z_{0} z_{b}}\left(z_{0}(p), z_{b}(p)\right)}
$$

on $C_{b}$ for $b=1, \ldots, M$, where the primes denote the derivatives with respect to $p$, then they act on $t_{\beta m}(m=0,1,2, \ldots)$ as

$$
\partial_{\alpha n} t_{\beta m}=\delta_{\alpha \beta} \delta_{n m}
$$

and on $v_{\beta m}(m=1,2, \ldots)$ as

$$
\partial_{\alpha n} v_{\beta m}= \begin{cases}-n m b_{\alpha n \beta m} & (n \neq 0) \\ -m b_{\alpha 0 \beta m} & (n=0)\end{cases}
$$

Remark 4.2. (47) implies that $t_{\alpha n}$ 's may be thought of as a system of local coordinates on $\mathcal{Z}$. (48) shows that the vector fields $\partial_{\alpha n}$ correspond to the time evolutions of the universal Whitham hierarchy.

Remark 4.3. $\partial_{\alpha n} z_{\beta}(p)$ 's are uniquely determined by (46). Though this is an implication of (47) and (48), one can directly confirm it as follows. Let $Z_{b}(p), Z_{0}(p)$ and $W_{b}(p)$ denote the three terms in (46). Consequently, they satisfy the equations

$$
Z_{b}(p)-Z_{0}(p)=W_{b}(p)
$$

for $b=1, \ldots, M$ in a neighborhood of $C_{b}$. As holomorphic functions, $Z_{b}(p), Z_{0}(p)$ are extended to $D_{b}$ and $\mathbf{C P}^{1} \backslash\left(D_{1} \cup \cdots \cup D_{M}\right)$ respectively, and behave as

$$
Z_{b}(p)=O(1) \quad\left(p \rightarrow q_{b}\right), \quad Z_{0}(p)=O\left(p^{-1}\right) \quad(p \rightarrow \infty) .
$$


One can decompose $Z_{0}(p)$ in $\mathbf{C P}^{1} \backslash\left(D_{1} \cup \cdots \cup D_{M}\right)$ as

$$
Z_{0}(p)=\sum_{a=1}^{M} Z_{0 a}(p), \quad Z_{0 a}(p)=\frac{1}{2 \pi i} \oint_{C_{a}} \frac{Z_{0}(q)}{q-p} d q
$$

$Z_{0 a}(p)$ is a holomorphic function in $\mathbf{C P}^{1} \backslash D_{a}, O\left(p^{-1}\right)$ as $p \rightarrow \infty$, and can be continued to a neighborhood of $C_{a}$ by deforming the contour $C_{a}$ inward. The foregoing equation for $Z_{b}(p)$ and $Z_{0}(p)$ can be thereby rewritten as

$$
\left(Z_{b}(p)-\sum_{a \neq b} Z_{0 a}(p)\right)-Z_{0 b}(p)=W_{b}(p) .
$$

One can consider this equation as splitting $W_{b}(p)$ into a sum of holomorphic functions $W_{b+}(p)$ and $W_{b-}(p)$ defined in $D_{b}$ and in $\mathbf{C P}^{1} \backslash D_{b}$, respectively. In particular,

$$
Z_{0 b}(p)=-W_{b-}(p)=-\frac{1}{2 \pi i} \oint_{C_{b}} \frac{W_{b}(q)}{p-q} d q \quad\left(p \in \mathbf{C P}^{1} \backslash D_{b}\right),
$$

hence

$$
Z_{0}(p)=-\sum_{a=1}^{M} \frac{1}{2 \pi i} \oint_{C_{a}} \frac{W_{a}(q)}{p-q} d q \quad\left(p \in \mathbf{C P}^{1} \backslash\left(D_{1} \cup \cdots \cup D_{M}\right)\right) .
$$

One can find a similar integral formula for $Z_{b}(p)$ as well.

Proof of Theorem 4.1. Let us first consider the action of $\partial_{\alpha n}$ on

$$
t_{0 m}=\sum_{b=1}^{M} \frac{1}{2 \pi i m} \oint_{C_{b}} H_{b, z_{0}}\left(z_{0}(p), z_{b}(p)\right) z_{0}(p)^{-m} z_{0}^{\prime}(p) d p .
$$

Applying $\partial_{\alpha n}$ to the integrand, we have the identity

$$
\begin{aligned}
& \partial_{\alpha n}\left(H_{b, z_{0}}\left(z_{0}(p), z_{b}(p)\right) z_{0}(p)^{-m} z_{0}^{\prime}(p)\right) \\
& =\frac{\partial}{\partial p}\left(H_{b, z_{0}}\left(z_{0}(p), z_{b}(p)\right) z_{0}(p)^{-m} \partial_{\alpha n} z_{0}(p)\right) \\
& \quad+H_{b, z_{0} z_{b}}\left(z_{0}(p), z_{b}(p)\right) z_{0}^{\prime}(p) z_{b}^{\prime}(p)\left(\frac{\partial_{\alpha n} z_{b}(p)}{z_{b}^{\prime}(p)}-\frac{\partial_{\alpha n} z_{0}(p)}{z_{0}^{\prime}(p)}\right) z_{0}(p)^{-m} \\
& =\frac{\partial}{\partial p}\left(H_{b, z_{0}}\left(z_{0}(p), z_{b}(p)\right) z_{0}(p)^{-m} \partial_{\alpha n} z_{0}(p)\right)+\Omega_{\alpha n}^{\prime}(p) z_{0}(p)^{-m} .
\end{aligned}
$$


Note that we have used the assumed equation (46) in the last line. Consequently,

$$
\begin{aligned}
\partial_{\alpha n} t_{0 m} & =\sum_{b=1}^{M} \frac{1}{2 \pi i m} \oint_{C_{b}} \Omega_{\alpha n}^{\prime}(p) z_{0}(p)^{-m} d p \\
& =\frac{1}{2 \pi i m} \oint_{C_{\infty}} \Omega_{\alpha n}^{\prime}(p) z_{0}(p)^{-m} d p,
\end{aligned}
$$

by deforming $C_{b}$ 's to a simple closed curve $C_{\infty}$ encircling $p=\infty$.

On the other hand, one can deduce from (31) and the first and the second equations in (32) (and the symmetry of $b_{\alpha n \beta m}$ ) the following Laurent expansion of $\Omega_{\alpha n}(p)$ 's with respect to $z_{0}(p)$ :

$$
\begin{aligned}
& \Omega_{0 n}(p)=z_{0}(p)^{n}+\sum_{m=1}^{\infty} n b_{0 n 0 m} z_{0}(p)^{-m}, \\
& \Omega_{a n}(p)=\sum_{m=1}^{\infty} n b_{a n 0 m} z_{0}(p)^{-m} \quad(n \geq 1), \\
& \Omega_{a 0}(p)=-\log z_{0}(p)+\sum_{m=1}^{\infty} b_{a 00 m} z_{0}(p)^{-m} .
\end{aligned}
$$

Inserting the derivatives

$$
\begin{aligned}
& \Omega_{0 n}^{\prime}(p)=n z_{0}(p)^{n-1} z_{0}^{\prime}(p)-\sum_{m=1}^{\infty} n m b_{0 n 0 m} z_{0}(p)^{-m-1} z_{0}^{\prime}(p), \\
& \Omega_{a n}^{\prime}(p)=-\sum_{m=1}^{\infty} n m b_{a n 0 m} z_{0}(p)^{-m-1} z_{0}^{\prime}(p) \quad(n \geq 1), \\
& \Omega_{a 0}^{\prime}(p)=-\frac{z_{0}^{\prime}(p)}{z_{0}(p)}-\sum_{m=1}^{\infty} m b_{a 00 m} z_{0}(p)^{-m-1} z_{0}^{\prime}(p)
\end{aligned}
$$

into the contour integral, we readily obtain (47) for $\beta=0$.

In the same way, the action of $\partial_{\alpha n}$ on

$$
v_{0 m}=\sum_{b=1}^{M} \frac{1}{2 \pi i} \oint_{C_{b}} H_{b, z_{0}}\left(z_{0}(p), z_{b}(p)\right) z_{b}(p)^{m} z_{b}^{\prime}(p) d p
$$

can be expressed as

$$
\partial_{\alpha n} v_{0 m}=\frac{1}{2 \pi i} \oint_{C_{\infty}} \Omega_{\alpha n}^{\prime}(p) z_{0}(p)^{m} d p
$$


This contour integral, too, can be evaluated by the foregoing Laurent expansions of $\Omega_{\alpha n}^{\prime}(p)$. We can thus derive (48) for $\beta=0$.

Let us now consider the action of $\partial_{\alpha n}$ on

$$
\begin{aligned}
t_{b m} & =\frac{1}{2 \pi i m} \oint_{C_{b}} H_{b, z_{b}}\left(z_{0}(p), z_{b}(p)\right) z_{b}(p)^{-m} z_{b}^{\prime}(p) d p \\
t_{b 0} & =\frac{1}{2 \pi i} \int_{C_{b}} H_{b, z_{b}}\left(z_{0}(p), z_{b}(p)\right) z_{b}^{\prime}(p) d p \\
v_{b m} & =\frac{1}{2 \pi i} \int_{C_{b}} H_{b, z_{b}}\left(z_{0}(p), z_{b}(p)\right) z_{b}(p)^{m} z_{b}^{\prime}(p) d p
\end{aligned}
$$

As in the previous case, we can deduce that

$$
\begin{aligned}
\partial_{\alpha n} t_{b m} & =-\frac{1}{2 \pi i m} \oint_{C_{b}} \Omega_{\alpha n}^{\prime}(p) z_{b}(w)^{-m} d p, \\
\partial_{\alpha n} t_{b 0} & =-\frac{1}{2 \pi i} \oint_{C_{b}} \Omega_{\alpha n}^{\prime}(p) d p, \\
\partial_{\alpha n} v_{b m} & =-\frac{1}{2 \pi i} \oint_{C_{b}} \Omega_{\alpha n}^{\prime}(p) z_{b}(p)^{m} d p,
\end{aligned}
$$

We can now use the following Laurent expansion of $\Omega_{\alpha n}(p)$ 's with respect to $z_{b}(p)$ derived from (31) and the second, third and fourth equations of (32):

$$
\begin{aligned}
& \Omega_{0 n}(p)=\sum_{m=0}^{\infty} n b_{0 n b m} z_{b}(p)^{-m}, \\
& \Omega_{a n}(p)=\delta_{a b} z_{b}(p)^{n}+\sum_{m=0}^{\infty} n b_{a n b m} z_{b}(p)^{-m}, \quad(n \geq 1), \\
& \Omega_{a 0}(p)= \begin{cases}-\log \epsilon_{b a}+\sum_{m=0}^{\infty} b_{a 0 b m} z_{b}(p)^{-m} & (b \neq a), \\
\log z_{a}(p)+\sum_{m=0}^{\infty} b_{a 0 a m} z_{a}(p)^{-m} & (b=a) .\end{cases}
\end{aligned}
$$

Inserting their derivatives

$$
\begin{aligned}
& \Omega_{0 n}^{\prime}(p)=-\sum_{m=0}^{\infty} n m b_{0 n b m} z_{b}(p)^{-m-1} z_{b}^{\prime}(p) \\
& \Omega_{a n}^{\prime}(p)=\delta_{a b} n z_{b}(p)^{n-1} z_{b}^{\prime}(p)-\sum_{m=0}^{\infty} n m b_{a n b m} z_{b}(p)^{-m-1} z_{b}^{\prime}(p) \quad(n \geq 1) \\
& \Omega_{a 0}^{\prime}(p)=\delta_{a b} \frac{z_{b}^{\prime}(p)}{z_{b}(p)}-\sum_{m=0}^{\infty} m b_{a 0 b m} z_{b}(p)^{-m-1} z_{b}^{\prime}(p)
\end{aligned}
$$

into the contour integrals (53), we can confirm the remaining parts of (47) and (48). This completes the proof of the theorem. 


\section{Construction of $\phi_{a}$ 's}

We construct the Phi functions $\phi_{a}, a=1, \ldots, M$, as follows:

$$
\begin{aligned}
\phi_{a}=\sum_{b=1}^{M} t_{b 0} b_{a 0 b 0}+\sum_{\gamma=0}^{M} \sum_{m=1}^{\infty} m t_{\gamma m} b_{a 0 \gamma m} & +\sum_{b=1}^{a-1} t_{b 0} \log (-1) \\
& -\frac{1}{2 \pi i} \sum_{b=1}^{M} \oint_{C_{b}} \frac{H_{b}\left(z_{0}(p), z_{b}(p)\right)}{p-q_{a}} d p .
\end{aligned}
$$

Proposition 5.1. The function $\phi_{a}$ defined by (55) satisfies

$$
\partial_{\beta n} \phi_{a}=\left\{\begin{array}{ll}
n b_{a 0 \beta n}, & (n \neq 0) \\
b_{a 0 \beta 0}+\log \epsilon_{a \beta}, & (n=0)
\end{array} .\right.
$$

Proof.

$$
\begin{aligned}
& \partial_{\beta n}\left(\frac{H_{b}\left(z_{0}(p), z_{b}(p)\right)}{p-q_{a}}\right) \\
&=\frac{H_{b, z_{0}}\left(z_{0}(p), z_{b}(p)\right)}{p-q_{a}} \partial_{\beta n} z_{0}(p)+\frac{H_{b, z_{b}}\left(z_{0}(p), z_{b}(p)\right)}{p-q_{a}} \partial_{\beta n} z_{b}(p) \\
&+\frac{H_{b}\left(z_{0}(p), z_{b}(p)\right)}{\left(p-q_{a}\right)^{2}} \partial_{\beta n} q_{a} .
\end{aligned}
$$

Therefore,

$$
\partial_{\beta n} \phi_{a}=T_{1}(\beta, n)+T_{2}(\beta, n)+ \begin{cases}n b_{a 0 \beta n}, & (n \neq 0) \\ b_{a 0 \beta 0}+\log \epsilon_{a \beta}, & (n=0)\end{cases}
$$

where

$$
\begin{aligned}
T_{1}(\beta, n) & =\sum_{b=1}^{M} t_{b 0} \partial_{\beta n} b_{a 0 b 0}+\sum_{\gamma=0}^{M} \sum_{m=1}^{\infty} m t_{\gamma m} \partial_{\beta n} b_{\gamma m a 0} \\
& =\sum_{m=1}^{\infty} m t_{0 m} \partial_{\beta n} b_{0 m a 0}+\sum_{b=1}^{M}\left(t_{b 0} \partial_{\beta n} b_{a 0 b 0}+\sum_{m=1}^{\infty} m t_{b m} \partial_{\beta n} b_{b m a 0}\right)
\end{aligned}
$$

and

$$
\begin{aligned}
T_{2}(\beta, n)=- & \frac{1}{2 \pi i} \sum_{b=1}^{M} \oint_{C_{b}}\left(\frac{H_{b, z_{0}}\left(z_{0}(p), z_{b}(p)\right)}{p-q_{a}} \partial_{\beta n} z_{0}(p)\right. \\
& \left.+\frac{H_{b, z_{b}}\left(z_{0}(p), z_{b}(p)\right)}{p-q_{a}} \partial_{\beta n} z_{b}(p)+\frac{H_{b}\left(z_{0}(p), z_{b}(p)\right)}{\left(p-q_{a}\right)^{2}} \partial_{\beta n} q_{a}\right) d p .
\end{aligned}
$$


The goal is to show that $T_{1}(\beta, n)+T_{2}(\beta, n)=0$ for all $(\beta, n)$. By the definition of $t_{0 m}$, we have

$$
\begin{aligned}
& \sum_{m=1}^{\infty} m t_{0 m} \partial_{\beta n} b_{0 m a 0} \\
& \quad=\frac{1}{2 \pi i} \sum_{b=1}^{M} \oint_{C_{b}} H_{b, z_{0}}\left(z_{0}(p), z_{b}(p)\right) z_{0}^{\prime}(p)\left(\sum_{m=1}^{\infty} \partial_{\beta n} b_{0 m a 0} z_{0}(p)^{-m}\right) d p .
\end{aligned}
$$

Differentiating

$$
-\log \left(p-q_{a}\right)=\Omega_{a 0}(p)=-\log z_{0}(p)+\sum_{m=1}^{\infty} b_{0 m a 0} z_{0}(p)^{-m}
$$

with respect to $t_{\beta n}$, we have

$$
\begin{aligned}
\frac{1}{p-q_{a}} \partial_{\beta n} q_{a}= & \sum_{m=1}^{\infty} \partial_{\beta n} b_{0 m a 0} z_{0}(p)^{-m} \\
& -\left(\frac{1}{z_{0}(p)}+\sum_{m=1}^{\infty} m b_{0 m a 0} z_{0}(p)^{-m-1}\right) \partial_{\beta n} z_{0}(p) \\
= & \sum_{m=1}^{\infty} \partial_{\beta n} b_{0 m a 0} z_{0}(p)^{-m}-\frac{1}{z_{0}^{\prime}(p)} \frac{1}{p-q_{a}} \partial_{\beta n} z_{0}(p)
\end{aligned}
$$

Therefore,

$$
\sum_{m=1}^{\infty} \partial_{\beta n} b_{0 m a 0} z_{0}(p)^{-m}=\frac{1}{p-q_{a}} \partial_{\beta n} q_{a}+\frac{1}{z_{0}^{\prime}(p)} \frac{1}{p-q_{a}} \partial_{\beta n} z_{0}(p),
$$

and

$$
\begin{aligned}
& \sum_{m=1}^{\infty} m t_{0 m} \partial_{\beta n} b_{0 m a 0}= \frac{1}{2 \pi i} \sum_{b=1}^{M} \oint_{C_{b}} H_{b, z_{0}}\left(z_{0}(p), z_{b}(p)\right) z_{0}^{\prime}(p) \times \\
& \times\left(\frac{1}{p-q_{a}} \partial_{\beta n} q_{a}+\frac{1}{z_{0}^{\prime}(p)} \frac{1}{p-q_{a}} \partial_{\beta n} z_{0}(p)\right) d p \\
&= \frac{1}{2 \pi i} \sum_{b=1}^{M} \oint_{C_{b}}\left(\frac{H_{b, z_{0}}\left(z_{0}(p), z_{b}(p)\right) z_{0}^{\prime}(p)}{p-q_{a}} \partial_{\beta n} q_{a}\right. \\
&\left.+\frac{H_{b, z_{0}}\left(z_{0}(p), z_{b}(p)\right)}{p-q_{a}} \partial_{\beta n} z_{0}(p)\right) d p
\end{aligned}
$$


In a similar way, the definition of $t_{b m}$ gives

$$
\begin{aligned}
t_{b 0} \partial_{\beta n} b_{a 0 b 0} & +\sum_{m=1}^{\infty} m t_{b m} \partial_{\beta n} b_{b m a 0} \\
& =\frac{1}{2 \pi i} \oint_{C_{b}} H_{b, z_{b}}\left(z_{0}(p), z_{b}(p)\right) z_{b}^{\prime}(p)\left(\sum_{m=0}^{\infty} \partial_{\beta n} b_{b m a 0} z_{b}(p)^{-m}\right) d p .
\end{aligned}
$$

Differentiating

$$
-\log \left(p-q_{a}\right)=\Omega_{a 0}(p)=\delta_{a b} \log z_{b}(p)+\sum_{m=0}^{\infty} b_{b m a 0} z_{b}(p)^{-m}-\log \epsilon_{b a}
$$

with respect to $t_{\beta n}$ and comparing it with $\Omega_{a 0}^{\prime}(p)$, we find that

$$
\frac{1}{p-q_{a}} \partial_{\beta n} q_{a}=\sum_{m=0}^{\infty} \partial_{\beta n} b_{b m a 0} z_{b}(p)^{-m}-\frac{1}{z_{b}^{\prime}(p)} \frac{1}{p-q_{a}} \partial_{\beta n} z_{b}(p) .
$$

Therefore,

$$
\begin{aligned}
& t_{b 0} \partial_{\beta n} b_{a 0 b 0}+\sum_{m=1}^{\infty} m t_{b m} \partial_{\beta n} b_{b m a 0} \\
= & \frac{1}{2 \pi i} \oint_{C_{b}}\left(\frac{H_{b, z_{b}}\left(z_{0}(p), z_{b}(p)\right) z_{b}^{\prime}(p)}{p-q_{a}} \partial_{\beta n} q_{a}+\frac{H_{b, z_{b}}\left(z_{0}(p), z_{b}(p)\right)}{p-q_{a}} \partial_{\beta n} z_{b}(p)\right) d p .
\end{aligned}
$$

Therefore,

$$
\begin{aligned}
& T_{1}(\beta, n)+T_{2}(\beta, n) \\
= & \frac{1}{2 \pi i} \sum_{b=1}^{M} \oint_{C_{b}}\left(\frac{H_{b, z_{0}}\left(z_{0}(p), z_{b}(p)\right) z_{0}^{\prime}(p)}{p-q_{a}} \partial_{\beta n} q_{a}\right. \\
& \left.\quad+\frac{H_{b, z_{b}}\left(z_{0}(p), z_{b}(p)\right) z_{b}^{\prime}(p)}{p-q_{a}} \partial_{\beta n} q_{a}-\frac{H_{b}\left(z_{0}(p), z_{b}(p)\right)}{\left(p-q_{a}\right)^{2}} \partial_{\beta n} q_{a}\right) d p \\
= & \frac{1}{2 \pi i} \sum_{b=1}^{M} \oint_{C_{b}} \frac{\partial}{\partial p}\left(\frac{H_{b}\left(z_{0}(p), z_{b}(p)\right)}{p-q_{a}}\right) d p \times \partial_{\beta n} q_{a}=0 .
\end{aligned}
$$

This completes the proof. 
Define

$$
\begin{aligned}
v_{a 0}= & -\phi_{a}+\sum_{b=1}^{a-1} t_{b 0} \log (-1) \\
= & -\sum_{b=1}^{M} t_{b 0} b_{a 0 b 0}-\sum_{\gamma=0}^{M} \sum_{m=1}^{\infty} m t_{\gamma m} b_{a 0 \gamma m} \\
& +\frac{1}{2 \pi i} \sum_{b=1}^{M} \oint_{C_{b}} \frac{H_{b}\left(z_{0}(p), z_{b}(p)\right)}{p-q_{a}} d p .
\end{aligned}
$$

Then (56) implies that

$$
\partial_{\beta n} v_{a 0}=\left\{\begin{array}{ll}
-n b_{a 0 \beta n}, & (n \neq 0), \\
-b_{a 0 \beta 0}, & (n=0)
\end{array} .\right.
$$

\section{Construction of the free energy $F$}

Let $J_{a, 1}\left(z_{0}, z_{a}\right)$ and $J_{a, 2}\left(z_{0}, z_{a}\right)$ be defined so that

$$
-\partial_{z_{a}} J_{a, 1}\left(z_{0}, z_{a}\right)=\partial_{z_{0}} J_{a, 2}\left(z_{0}, z_{a}\right)=H_{a}\left(z_{0}, z_{a}\right) H_{a, z_{0} z_{a}}\left(z_{0}, z_{a}\right) .
$$

We construct the $F$ function as follows:

$$
\begin{aligned}
F= & \frac{1}{2} \sum_{a=1}^{M} t_{a 0} v_{a 0}+\frac{1}{2} \sum_{\alpha=0}^{M} \sum_{n=1}^{\infty} t_{\alpha n} v_{\alpha n} \\
& +\frac{1}{8 \pi i} \sum_{a=1}^{M} \oint_{C_{a}}\left\{J_{a, 1}\left(z_{0}(p), z_{a}(p)\right) z_{0}^{\prime}(p)+J_{a, 2}\left(z_{0}(p), z_{a}(p)\right) z_{a}^{\prime}(p)\right\} d p .
\end{aligned}
$$

Proposition 6.1. The F function defined by (60) satisfies

$$
\partial_{\beta n} F=v_{\beta n}
$$


Proof. A direct computation shows that

$$
\begin{aligned}
& \partial_{\beta n}\left\{J_{a, 1}\left(z_{0}(p), z_{a}(p)\right) z_{0}^{\prime}(p)+J_{a, 2}\left(z_{0}(p), z_{a}(p)\right) z_{a}^{\prime}(p)\right\} \\
= & \frac{\partial}{\partial p}\left\{J_{a, 1}\left(z_{0}(p), z_{a}(p)\right) \partial_{\beta n} z_{0}+J_{a, 2}\left(z_{0}(p), z_{a}(p)\right) \partial_{\beta n} z_{a}\right\} \\
& -2 H_{a}\left(z_{0}(p), z_{a}(p)\right)\left(\partial_{\beta n} z_{a}(p) z_{0}^{\prime}(p)-z_{a}^{\prime}(p) \partial_{\beta n} z_{0}(p)\right) \\
= & \frac{\partial}{\partial p}\left\{J_{a, 1}\left(z_{0}(p), z_{a}(p)\right) \partial_{\beta n} z_{0}+J_{a, 2}\left(z_{0}(p), z_{a}(p)\right) \partial_{\beta n} z_{a}\right\} \\
& -2 H_{a}\left(z_{0}(p), z_{a}(p)\right) \Omega_{\beta n}^{\prime}(p)
\end{aligned}
$$

by using the definition of the vector field (46). Hence,

$$
\partial_{\beta n} F=I_{1}(\beta, n)+I_{2}(\beta, n) .
$$

where

$$
\begin{aligned}
& I_{1}(\beta, n)=\frac{v_{\beta n}}{2}+\frac{1}{2} \sum_{a=1}^{M} t_{a 0} \partial_{\beta n} v_{a 0}+\frac{1}{2} \sum_{\alpha=0}^{M} \sum_{m=1}^{\infty} t_{\alpha m} \partial_{\beta n} v_{\alpha m}, \\
& I_{2}(\beta, n)=-\frac{1}{4 \pi i} \sum_{a=1}^{M} \oint_{C_{a}} H_{a}\left(z_{0}(p), z_{a}(p)\right) \Omega_{\beta n}^{\prime}(p) d p .
\end{aligned}
$$

Now if $n \neq 0$, integration by parts shows that

$$
\begin{aligned}
I_{2}(\beta, n)= & -\frac{1}{4 \pi i} \sum_{a=1}^{M} \oint_{C_{a}} H_{a}\left(z_{0}(p), z_{a}(p)\right) \Omega_{\beta n}^{\prime}(p) d p \\
= & \frac{1}{4 \pi i} \sum_{a=1}^{M} \oint_{C_{a}} H_{a, z_{0}}\left(z_{0}(p), z_{a}(p)\right) z_{0}^{\prime}(p) \Omega_{\beta n}(p) d p \\
& +\frac{1}{4 \pi i} \sum_{a=1}^{M} \oint_{C_{a}} H_{a, z_{a}}\left(z_{0}(p), z_{a}(p)\right) z_{a}^{\prime}(p) \Omega_{\beta n}(p) d p .
\end{aligned}
$$


If $\beta=0$, the first equation in (52) and the first equation in (54) show that

$$
\begin{aligned}
I_{2}(0, n)= & \frac{1}{4 \pi i} \sum_{a=1}^{M} \oint_{C_{a}} H_{a, z_{0}}\left(z_{0}(p), z_{a}(p)\right) z_{0}^{\prime}(p) \times \\
& \times\left(z_{0}(p)^{n}+n \sum_{m=1}^{\infty} b_{0 m 0 n} z_{0}(p)^{-m}\right) d p \\
& +\frac{1}{4 \pi i} \sum_{a=1}^{M} \oint_{C_{a}} H_{a, z_{a}}\left(z_{0}(p), z_{a}(p)\right) z_{a}^{\prime}(p) \times \\
& \times\left(n \sum_{m=0}^{\infty} b_{0 n a m} z_{a}(p)^{-m}\right) d p \\
= & \frac{v_{0 n}}{2}+\frac{1}{2} \sum_{m=1}^{\infty} n m b_{0 m 0 n} t_{0 m}+\frac{1}{2} \sum_{a=1}^{M} n b_{0 n a 0} t_{a 0} \\
& +\frac{1}{2} \sum_{a=1}^{M} \sum_{m=1}^{\infty} n m b_{0 n a m} t_{a m} \\
= & \frac{v_{0 n}}{2}-\frac{1}{2} \sum_{a=1}^{M} t_{a 0} \partial_{0 n} v_{a 0}-\frac{1}{2} \sum_{\alpha=0}^{M} \sum_{m=1}^{\infty} t_{\alpha m} \partial_{0 n} v_{\alpha m},
\end{aligned}
$$

by the definition of $t_{\alpha n}$ (44, 45) and actions of $\partial_{\alpha n}$ on $v_{\beta m}$ (48, 58). Therefore,

$$
\partial_{0 n} F=I_{1}(0, n)+I_{2}(0, n)=v_{0 n} .
$$

If $\beta=b \neq 0, n \neq 0$, the second equation in (52) and the second equation in 
(54) show that

$$
\begin{aligned}
I_{2}(b, n)= & \frac{1}{4 \pi i} \sum_{a=1}^{M} \oint_{C_{a}} H_{a, z_{0}}\left(z_{0}(p), z_{a}(p)\right) z_{0}^{\prime}(p) \times \\
& \times\left(n \sum_{m=1}^{\infty} b_{b n 0 m} z_{0}(p)^{-m}\right) d p \\
& +\frac{1}{4 \pi i} \sum_{a=1}^{M} \oint_{C_{a}} H_{a, z_{a}}\left(z_{0}(p), z_{a}(p)\right) z_{a}^{\prime}(p) \times \\
& \times\left(\delta_{a b} z_{a}(p)^{n}+n \sum_{m=0}^{\infty} b_{b n a m} z_{a}(p)^{-m}\right) d p \\
= & \frac{1}{2} \sum_{m=0}^{\infty} n m b_{b n 0 m} t_{0 m}+\frac{v_{b n}}{2}+\frac{1}{2} \sum_{a=1}^{M} n b_{b n a 0} t_{a 0} \\
& +\frac{1}{2} \sum_{a=1}^{M} \sum_{m=1}^{\infty} n m b_{b n a m} t_{a m} \\
= & \frac{v_{b n}}{2}-\frac{1}{2} \sum_{a=1}^{M} t_{a 0} \partial_{b n} v_{a 0}-\frac{1}{2} \sum_{\alpha=0}^{M} \sum_{m=1}^{\infty} t_{\alpha m} \partial_{b n} v_{\alpha m}
\end{aligned}
$$

again by (44, 45) and (48, 58). Therefore,

$$
\partial_{b n} F=I_{1}(b, n)+I_{2}(b, n)=v_{b n} .
$$

Now if $\beta=b \neq 0, n=0$, we have

$$
\Omega_{b 0}^{\prime}(p)=-\frac{1}{p-q_{b}} .
$$

Therefore,

$$
\begin{aligned}
I_{2}(b, 0) & =-\frac{1}{4 \pi i} \sum_{a=1}^{M} \oint_{C_{a}} H_{a}\left(z_{0}(p), z_{a}(p)\right) \Omega_{b 0}^{\prime}(p) d p \\
& =\frac{1}{4 \pi i} \sum_{a=1}^{M} \oint_{C_{a}} \frac{H_{a}\left(z_{0}(p), z_{a}(p)\right)}{p-q_{b}} d p .
\end{aligned}
$$


On the other hand,

$$
\begin{aligned}
I_{1}(b, 0) & =\frac{v_{b 0}}{2}+\frac{1}{2} \sum_{a=1}^{M} t_{a 0} \partial_{b 0} v_{a 0}+\frac{1}{2} \sum_{\alpha=0}^{M} \sum_{m=1}^{\infty} t_{\alpha m} \partial_{b 0} v_{\alpha m} \\
& =\frac{v_{b 0}}{2}-\frac{1}{2} \sum_{a=1}^{M} t_{a 0} b_{a 0 b 0}-\frac{1}{2} \sum_{\alpha=0}^{M} \sum_{m=1}^{\infty} m t_{\alpha m} b_{\alpha m b 0},
\end{aligned}
$$

by (48, 58). Hence,

$$
\begin{aligned}
& I_{1}(b, 0)+I_{2}(b, 0) \\
= & \frac{v_{b 0}}{2}-\frac{1}{2} \sum_{a=1}^{M} t_{a 0} b_{a 0 b 0}-\frac{1}{2} \sum_{\alpha=0}^{M} \sum_{m=1}^{\infty} m t_{\alpha m} b_{\alpha m b 0} \\
& +\frac{1}{4 \pi i} \sum_{a=1}^{M} \oint_{C_{a}} \frac{H_{a}\left(z_{0}(p), z_{a}(p)\right)}{p-q_{b}} d p \\
= & \frac{v_{b 0}}{2}+\frac{v_{b 0}}{2}=v_{b 0}
\end{aligned}
$$

because of (57). This completes the proof.

This proposition and the definition of $v_{a 0}$ (57) shows that the $F$ function indeed satisfies (29).

\section{Special String Equations}

In this section, we consider the special case where the generating functions $H_{a}\left(z_{0}, z_{a}\right), a=1, \ldots, M$, have the form

$$
H_{a}\left(z_{0}, z_{a}\right)=z_{0}^{\nu_{0}} z_{a}^{\nu_{a}}, \quad \nu_{0}, \nu_{a} \in \mathbb{N},
$$

so that the string equations (40) become

$$
\begin{aligned}
& \zeta_{0}(p)=\nu_{0} z_{0}(p)^{\nu_{0}-1} z_{a}(p)^{\nu_{a}}, \\
& \zeta_{a}(p)=-\nu_{a} z_{0}(p)^{\nu_{0}} z_{a}(p)^{\nu_{a}-1}
\end{aligned}
$$

for $p \in C_{a}$. These string equations were discussed in [6]. ¿From (61) and (16), we have

$$
\begin{aligned}
\nu_{0} z_{0}(p)^{\nu_{0}-1} z_{a}(p)^{\nu_{a}} & =\sum_{n=1}^{\infty} n t_{0 n} z_{0}(p)^{n-1}+\frac{t_{00}}{z_{0}(p)}+\sum_{n=1}^{\infty} z_{0}(p)^{-n-1} v_{0 n}, \\
-\nu_{a} z_{0}(p)^{\nu_{0}} z_{a}(p)^{\nu_{a}-1} & =\sum_{n=1}^{\infty} n t_{a n} z_{a}(p)^{n-1}+\frac{t_{a 0}}{z_{a}(p)}+\sum_{n=1}^{\infty} z_{a}(p)^{-n-1} v_{a n}
\end{aligned}
$$


for $p \in C_{a}$. The definitions of $t_{\alpha n}$ and $v_{\alpha n}$ (44) and (45) then become

$$
\begin{aligned}
n t_{0 n} & =\sum_{a=1}^{M} \frac{\nu_{0}}{2 \pi i} \oint_{C_{a}} z_{0}(p)^{\nu_{0}-n-1} z_{a}(p)^{\nu_{a}} d z_{0}(p) \\
t_{00} & =\sum_{a=1}^{M} \frac{\nu_{0}}{2 \pi i} \oint_{C_{a}} z_{0}(p)^{\nu_{0}-1} z_{a}(p)^{\nu_{a}} d z_{0}(p), \\
v_{0 n} & =\sum_{a=1}^{M} \frac{\nu_{0}}{2 \pi i} \oint_{C_{a}} z_{0}(p)^{\nu_{0}+n-1} z_{a}(p)^{\nu_{a}} d z_{0}(p)
\end{aligned}
$$

and

$$
\begin{aligned}
n t_{a n} & =\frac{\nu_{a}}{2 \pi i} \oint_{C_{a}} z_{0}(p)^{\nu_{0}} z_{a}(p)^{\nu_{a}-n-1} d z_{a}(p), \\
t_{a 0} & =\frac{\nu_{a}}{2 \pi i} \oint_{C_{a}} z_{0}(p)^{\nu_{0}} z_{a}(p)^{\nu_{a}-1} d z_{a}(p), \\
v_{a n} & =\frac{\nu_{a}}{2 \pi i} \oint_{C_{a}} z_{0}(p)^{\nu_{0}} z_{a}(p)^{\nu_{a}+n-1} d z_{a}(p) .
\end{aligned}
$$

The functions $J_{a, 1}\left(z_{0}, z_{a}\right)$ and $J_{a, 2}\left(z_{0}, z_{a}\right)$ (59) can be chosen to be

$$
J_{a, 1}\left(z_{0}, z_{a}\right)=-\frac{\nu_{0}}{2} z_{0}^{2 \nu_{0}-1} z_{a}^{2 \nu_{a}}, \quad J_{a, 2}\left(z_{0}, z_{a}\right)=\frac{\nu_{a}}{2} z_{0}^{2 \nu_{0}} z_{a}^{2 \nu_{a}-1} .
$$

The free energy (60) then becomes

$$
\begin{aligned}
F= & \frac{1}{2} \sum_{a=1}^{M} t_{a 0} v_{a 0}+\frac{1}{2} \sum_{\alpha=0}^{M} \sum_{n=1}^{\infty} t_{\alpha n} v_{\alpha n} \\
& -\frac{\nu_{0}}{16 \pi i} \sum_{a=1}^{M} \oint_{C_{a}} z_{0}(p)^{2 \nu_{0}-1} z_{a}(p)^{2 \nu_{a}} d z_{0}(p) \\
& +\frac{1}{16 \pi i} \sum_{a=1}^{M} \oint_{C_{a}} \nu_{a} z_{0}(p)^{2 \nu_{0}} z_{a}(p)^{2 \nu_{a}-1} d z_{a}(p)
\end{aligned}
$$


Using (62) and (63), we find that

$$
\begin{aligned}
& -\frac{\nu_{0}}{16 \pi i} \sum_{a=1}^{M} \oint_{C_{a}} z_{0}(p)^{2 \nu_{0}-1} z_{a}(p)^{2 \nu_{a}} d z_{0}(p) \\
= & -\frac{1}{16 \pi i} \sum_{a=1}^{M} \oint_{C_{a}}\left(\nu_{0} z_{0}(p)^{\nu_{0}-1} z_{a}(p)^{\nu_{a}}\right) z_{0}(p)^{\nu_{0}} z_{a}(p)^{\nu_{a}} d z_{0}(p) \\
= & -\frac{1}{16 \pi i} \sum_{a=1}^{M} \oint_{C_{a}}\left(\sum_{n=1}^{\infty} n t_{0 n} z_{0}(p)^{n-1}+\frac{t_{00}}{z_{0}(p)}+\sum_{n=1}^{\infty} z_{0}(p)^{-n-1} v_{0 n}\right) \\
= & -\frac{1}{8 \nu_{0}}\left(2 \sum_{n=1}^{\infty} n t_{0 n} v_{0 n}+t_{00}^{2}\right) .
\end{aligned}
$$

Similarly, one can show that

$$
\frac{1}{16 \pi i} \oint_{C_{a}} \nu_{a} z_{0}(p)^{2 \nu_{0}} z_{a}(p)^{2 \nu_{a}-1} d z_{a}(p)=-\frac{1}{8 \nu_{a}}\left(2 \sum_{n=1}^{\infty} n t_{a n} v_{a n}+t_{a 0}^{2}\right) .
$$

Therefore, the free energy is given explicitly by

$$
F=-\frac{1}{8}\left(\frac{t_{00}^{2}}{\nu_{0}}+\sum_{a=1}^{M} \frac{t_{a 0}^{2}}{\nu_{a}}\right)+\frac{1}{2} \sum_{a=1}^{M} t_{a 0} v_{a 0}+\frac{1}{2} \sum_{\alpha=0}^{M} \sum_{n=1}^{\infty}\left(1-\frac{n}{2 \nu_{\alpha}}\right) t_{\alpha n} v_{\alpha n} .
$$

\section{References}

[1] F. Guil, M. Mañas and L. Martínez Alonso, On the Whitham hierarchies: reductions and hodograph solutions, J. Phys. A36 (2003), 40474062 (arXiv:nlin/0209051).

[2] B. Konopelchenko and L. Martínez Alonso, $\bar{\partial}$-equations, integrable deformations of quasi-conformal mappings and Whitham hierarchy, Phys. Lett. A286 (2001), 161-166 (arXiv:nin.SI/0103015).

[3] B. Konopelchenko and L. Martínez Alonso, Dispersionless scalar integrable hierarchies, Whitham hierarchy and the quasiclassical $\bar{\partial}$-dressing method, J. Math. Phys. 43 (2001), 3807-3823 (arXiv:nlin.SI/0105071). 
[4] I.M. Krichever, The $\tau$-function of the universal Whitham hierarchy, matrix models and topological field theories, Comm. Pure. Appl. Math. 47 (1994), 437-475 (arXiv:hep-th/9205110).

[5] M. Mañas, E. Medina and L. Martínez Alonso, On the Whitham hierarchy: Dressing scheme, string equations and additional symmetries, J. Phys. A: Math. Gen. 39 (2006), 2349-2382 (arXiv:nlin-si/0509017).

[6] L. Martínez Alonso, E. Medina and M. Mañas, String equations in Whitham hierarchies: $\tau$-functions and Virasoro constraints, Comm. Math. Phys. 274 (2007), 627-658 (arXiv:nlin-si/0510001).

[7] L. Martínez Alonso and E. Medina, Multiple orthogonal polynomials, string equations and the large- $\boldsymbol{n}$ limit, J. Phys. A: Math. Theor. 42 (2009), 205204 (arXiv:0812.3817 [nlin.SI]).

[8] K. Takasaki, Dispersionless Toda hierarchy and two-dimensional string theory, Commun. Math. Phys. 170 (1995), 101-116 (arXiv:hep-th/9403190).

[9] K. Takasaki and T. Takebe, Integrable hierarchies and dispersionless limit, Rev. Math. Phys. 7 (1995), 743-808 (arXiv:hep-th/9405096)

[10] K. Takasaki and T. Takebe, Universal Whitham hierarchy, dispersionless Hirota equations and multicomponent KP hierarchy, Physica D235 (2007), 109-125 (arXiv:nlin-si/0608068).

[11] K. Takasaki and T. Takebe, Loewner equations, Hirota equations and reductions of universal Whitham hierarchy, J. Phys. A: Math. Theor. 41 (2008), 475206 (27pp) (arXiv:0808.1444 [nlin.SI]).

[12] L.P. Teo, Conformal mappings and dispersionless Toda hierarchy, Commun. Math. Phys. 292 (2009), 391-415 (arXiv:0905.3599 [math-ph]).

[13] L.P. Teo, Conformal mappings and dispersionless Toda hierarchy II: General string equations, arXiv:0906.3565 [math-ph].

[14] P.B. Wiegmann and A. Zabrodin, Conformal maps and integrable hierarchies, Comm. Math. Phys. 213 (2000), 523-538 (arXiv:hep-th/9909147).

[15] A. Zabrodin, Dispersionless limit of Hirota equations in some problems of complex analysis, Theor. Math. Phys. 12 (2001), 1511-1525 (arXiv:math/0104169). 